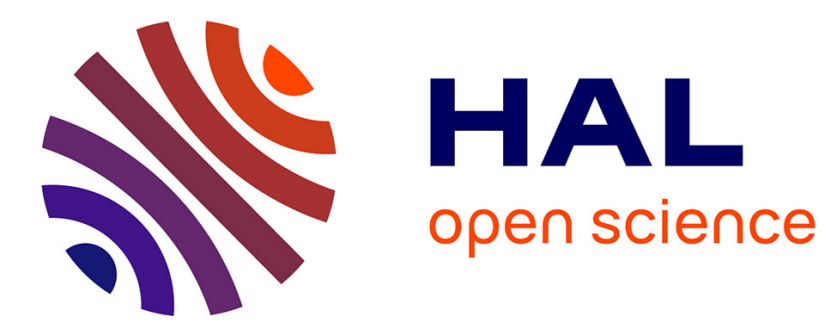

\title{
The Cosmological constant problem
}

Philippe Brax

\section{To cite this version:}

Philippe Brax. The Cosmological constant problem. Contemporary Physics, 2004, 45, pp.227-236. 10.1080/00107510410001662286 . hal-00165345

\section{HAL Id: hal-00165345 \\ https://hal.science/hal-00165345}

Submitted on 25 Jul 2007

HAL is a multi-disciplinary open access archive for the deposit and dissemination of scientific research documents, whether they are published or not. The documents may come from teaching and research institutions in France or abroad, or from public or private research centers.
L'archive ouverte pluridisciplinaire HAL, est destinée au dépôt et à la diffusion de documents scientifiques de niveau recherche, publiés ou non, émanant des établissements d'enseignement et de recherche français ou étrangers, des laboratoires publics ou privés. 


\title{
The Cosmological Constant Problem
}

\author{
Ph. $\operatorname{Brax}^{1 a}$ \\ a Service de Physique Théorique \\ $C E A / D S M / S P h T$, Unité de recherche associée au CNRS, \\ CEA-Saclay F-91191 Gif/Yvette cedex, France
}

\begin{abstract}
Observational evidence seems to indicate that the expansion of the universe is currently accelerating. Such an acceleration strongly suggests that the content of the universe is dominated by a non-clustered form of matter, the so-called dark energy. The cosmological constant, introduced by Einstein to reconcile General Relativity with a closed and static Universe, is the most likely candidate for dark energy although other options such as a weakly interacting field, also known as quintessence, have been proposed. The fact that the dark energy density is some one hundred and twenty orders of magnitude lower than the energy scales present in the early universe constitutes the cosmological constant problem. We review various aspects of the cosmological constant problem and some interesting scenarios using supersymmetry or extra-dimensions attempting to solve one of the most puzzling issues in physics.
\end{abstract}

\footnotetext{
${ }^{1}$ brax@spht.saclay.cea.fr
} 


\section{The Cosmological Constant}

It is now common knowledge that the Universe is expanding. More puzzling is the recent evidence that this expansion is accelerating. Not only distant galaxies are receding away from us, they do so in an accelerating fashion. The quest for an explanation of the acceleration of the Universe is still in its infancy. Many scenarios have been proposed. No complete understanding has been reached yet. The purpose of this introductory review is to present parts of the observational evidence and some of the theoretical models which have been proposed. More details and a comprehensive list of references can be found in the reviews $[1,2,3,4,5]$. The figures have been taken from the research articles $[6,7,8,9]$.

The dynamics of the Universe are described by the general theory of relativity. In general relativity, matter and geometry are intrinsically entangled. Space-time is curved by matter as well-known from black-hole physics where the gravitational effects are so strong that even light cannot escape to infinity. The Einstein equations relate on the one hand geometry, in the form of the Ricci tensor $R_{\mu \nu}$, the metric tensor $g_{\mu \nu}$ and the curvature $R$, and matter represented by the energy-momemtum tensor $T_{\mu \nu}$

$$
R_{\mu \nu}-\frac{1}{2} g_{\mu \nu} R=-8 \pi G_{N} T_{\mu \nu}
$$

where $G_{N}$ is Newton's constant of gravitation. The Ricci tensor measures the curvature of space-time while the energy-momentum tensor embodies the effects of pressure and energy. These equations relate tensors, here four by four matrices, leading to highly non-linear equations. All gravitational phenomena are governed by Einstein equations. In particular we will be interested in cosmological features occurring on time-scales of the order of the age of the Universe and on distance scales as large as the size of the Universe. Even on such extraordinary scales, general relativity is thought to apply and govern all cosmological phenomena. When trying to analyse the cosmology of galaxies and galaxy clusters, it is convenient to model the behaviour of matter with only two quantities: energy density and pressure. This is enough to characterise dust-like matter as well as radiation, two of the key components of our Universe. For ordinary matter and radiation, the energy momentum tensor is diagonal and determined by the energy density $\rho$ measured in units of energy per unit volume and $p$ the pressure. All energy scales will be measured in units where $\hbar=c=1$. This implies that length scales can be converted into inverse energy scales, therefore energy densities will be expressed in electron-Volt to the fourth power.

In 1917, Einstein applied his equations to cosmology. Lacking the observational evidence on the recession of galaxies (discovered by E. Hubble in 1929), Einstein assumed that the Universe was closed and static. A closed universe is such that space is spherical, i.e. spatially finite with no boundary. A static Universe is such that its shape is time-independent. Unfortunately these two hypotheses are incompatible with Einstein's equations. In order to accommodate a closed and static Universe, Einstein modified his equations by introducing 
a new term: the cosmological constant $\Lambda$

$$
R_{\mu \nu}-\frac{1}{2} g_{\mu \nu} R=-8 \pi G_{N} T_{\mu \nu}+\Lambda g_{\mu \nu}
$$

The positive cosmological constant $\Lambda$ introduces a repulsive effect which compensates the gravitational pull. A closed and static Universe is a solution of the modified Einstein equations with a radius $\sqrt{\frac{3}{\Lambda}}$. The cosmological constant $\Lambda$ has dimension two in energy unit. It can be interpreted as another source of matter with a diagonal energy momentum-tensor (as for dust-like matter and radiation). The pressure due to a cosmological constant is the opposite of the energy density corresponding to a so-called equation of state $w=-1$. The equation of state in statistical mechanics is the relation between pressure and energy density. In cosmology it is common practise to call the equation of state the proportionality coefficient relating pressure and density. The repulsive nature of the cosmological constant is due to a negative pressure. We will encounter other types of matter with the same kind of repulsiveness when discussing scalar fields.

After the discovery of the galaxy recession, the necessity of the cosmological constant became doubtful. Indeed Friedmann and then independently Lemaitre showed that the Hubble flow of galaxies could be explained within general relativity without the need for a cosmological constant. Until recently the cosmological constant was deemed unphysical as can be read in the highly influential textbook on the classical theory of fields by Landau and Lifschitz.

Since the early eighties, the inflationary paradigm has reinstated the cosmological constant at the forefront of current research. Indeed the standard cosmological model relying on the homogeneity, i.e. the Copernican principle that no particular place in the Universe plays a special role, and the isotropy of the Universe, i.e. on large scales there is no preferred direction, suffers from many problems, among others the horizon problem. As the velocity of light is finite, how come that regions of space which were not in causal contact in the past look so similar now? In particular how come that the temperature of the cosmic microwave background, the remnant radiation from the very early Universe is so isotropic? This can be explained using inflation, a period of exponentially fast expansion in the early Universe. When inflating, regions in causal contact are blown up so fast as to become acausal.

So what is the connection between inflation and the cosmological constant? Inflation can be understood as driven by a constant energy density acting as a cosmological constant. This can be easily grasped by looking at one of the most important equations of cosmology: the Friedmann equation

$$
H^{2}=8 \pi G_{N} \frac{\rho}{3}+\frac{\Lambda}{3}-\frac{k}{a^{2}}
$$

Here $a$ is the cosmic scale factor measuring the size of the Universe and $H=\frac{\dot{a}}{a}$ is the Hubble rate corresponding to the ratio between the speed of receding galaxies and their distance. In a closed and static Universe the Hubble rate 
vanishes $H=0$, a compensation due to the curvature term $k=1$ and the cosmological constant $\Lambda$. In a flat Universe $k=0$ with no cosmological constant $\Lambda=0$, the Hubble rate is non-vanishing due to the presence of matter $\rho$. In a flat Universe dominated by a constant cosmological constant $\Lambda$ the Hubble rate is constant. This is the de Sitter model leading to an exponential increase of the scale factor $a$. Primordial inflation in the early Universe is thought to occur due to a large energy density mimicking the presence of a cosmological constant. This is enough to create an enormous blowing up of distance scales. The end of inflation is due to the sudden transfer of the inflationary energy density to ordinary particles. The origin of this energy density is often modelled by a scalar field. This idea also reappears in models of quintessence as we will discuss shortly.

After inflation the Universe is radiation dominated and then matter dominated. In the matter dominated era, structures such as galaxies are formed due to the collapse of matter density fluctuations seeded by quantum fluctuations in the early universe. Well-known physics such as particle and nuclear physics constrain the evolution of the Universe since nucleosynthesis (when elements such as helium were created). In particular, it is observationally clear that a cosmological constant, if present, must be negligible from nucleosynthesis till the very recent past measured in redshift denoted by $z$ and defined by $a=\frac{a_{0}}{1+z}$ where $a_{0}$ is the scale factor of the Universe now, i.e. from a red-shift of a few thousand to one. The red-shit $z$ is observationally accessible from the displacement of the light spectra coming from distant objects compared to the light spectra measured on earth. In terms of age, a red-shift of unity corresponds to events occurring roughly at a third of the age of the Universe. In the recent past from a red-shift of order unity, it seems that the cosmological constant plays a significant role in the dynamics of the Universe.

\section{Observations and the Cosmological Constant}

In cosmology, empirical results have a different status from ordinary laboratory experiments. Indeed we have access to a single Universe and one cannot tamper with the fate of the Universe by changing parameters as one would do in a laboratory experiment. Moreover, observations of distant phenomena are plagued with uncertainty, known as systematics, due to our incomplete understanding of features such as the intergalactic medium. Another important source of uncertainty springs from the difficulty of measuring distances. All these issues are highly relevant when interpreting one of the major sources of information on the cosmological constant: the Hubble diagram as deduced from the explosion of type Ia supernovae. These supernovae are believed to result from the explosion of white dwarfs accreting neighbouring stars whose mass go above a threshold known as the Chandrasekar limit. Recently type Ia supernovae have been used as standard candles, i.e. they are assumed to be all of the same type from low red-shift to $z \sim 1$. This hypothesis is borne out by their supposed common origin. The intrinsic luminosity of type Ia supernovae is considered 
to be constant with $M \sim-19.5$, as bright as the whole galaxy they inhabit. Distances are measured by astronomers using the magnitude difference $m-M$ where $m$ is the apparent magnitude and $M$ the absolute magnitude of an object. This difference is nothing but the logarithm of the luminosity distance obtained by using the inverse square decrease of the measured flux of light received on earth compared to the intrinsic luminosity of a distant object. The apparent magnitude of type Ia supernovae can be inferred as a function of the red-shift $z$. The luminosity distance depends on the geometry of the Universe. At small red-shift, the luminosity distance is proportional to the red-shift of an object. For more distant objects, this linear relation is distorted when the Universe accelerates. Hence, the measurement of the luminosity distance gives a direct clue about the acceleration of the Universe. The quadratic correction the linear relationship between the red-shift and the luminosity distance depends on the acceleration parameter $q_{0}$ now. A non-vanishing and negative $q_{0}$ indicates that not only the Universe is expanding but the expansion itself is accelerating. It seems that, as seen in figure 1 , this is the case. Possible sources of uncertainty could be an intrinsic difference between supernovae at different red-shifts and extinction of the light received on earth due to the intergalactic dust.

The acceleration parameter is crucially related to the matter content of the universe $q_{0}=\frac{\Omega_{m}}{2}-\Omega_{\Lambda}$ where $\Omega_{m}$ is the matter fraction and $\Omega_{\Lambda}$ is the cosmological constant fraction. The energy fraction $\Omega_{i}$ is the ratio of the energy density of a species $i$ to the critical density of the Universe $\rho_{c}$. The critical energy density of the Universe is the energy density which would lead to the present Hubble rate, i.e. would explain the recession of galaxies, in a flat Universe with no cosmological constant or dark energy. It has been measured to be $\rho_{c} \approx 10^{-48} \mathrm{GeV}^{4}$. Measurements of $q_{0}$ give access to one relation between the cosmological constant and matter fractions. Another constraint is provided by the cosmic microwave background.

The temperature anisotropies in the cosmic microwave background allows one to constrain the cosmological fractions. These anisotropies are one of the benchmarks of modern cosmology. Their origin gives us a hint about the nature of the early universe. The cosmic microwave background was discovered in 1965 by Penzias and Wilson. It corresponds to a bath of radiation at the very low temperature of $2.7 \mathrm{~K}$. It appears to be completely isotropic, i.e. photons arising from all directions in the sky have the same temperature. It is only in 1992 that the satellite COBE measured fluctuations in temperature of the order of $10^{-5}$. Such small fluctuations are not uniformly distributed on the sky, they can be decomposed into the spherical harmonics - like the eigenstates of the hydrogen atom. The origin of these fluctuations is thought to be due to quantum fluctuations in the early universe. These quantum fluctuations result in radiation energy density fluctuations. When matter and radiation decouple around $z=1300$, at the last scattering surface, the photons are then nearly free. These photons, red-shifted by the expansion of the Universe, are the ones composing the cosmic microwave radiation. Small fluctuations in the radiation energy density at the last scattering surface appear to us as small temperature fluctuations. These fluctuations are are random variables which are easier to 
characterise using their power spectrum $C_{l}$ depending only on the multipole number $l$. The statistical average is taken over the possible realisations of the random processes creating the primordial fluctuations. Of course we have only one realization (our Universe) so observationally one has to resort to the ergodic hypothesis according to which the statistical average and the spatial average over the sky are equal.

The latest results on the $C_{l}$ spectrum have been obtained by the WMAP experiment. In particular a structure of acoustic peaks is clearly observed. These peaks correspond to the wave patterns of the temperature fluctuations across the sky. Small $l$ is equivalent to large structures whereas large $l$ probes small structures. One prominent feature of the $C_{l}$ spectrum is the existence of a large first peak whose location is related to the total matter content of the universe $\Omega_{0}=\Omega_{m}+\Omega_{\Lambda}$ by $l_{\text {peak }} \sim 220 \Omega_{0}^{-1 / 2}$.

The measurement of the first peak location confirms that our Universe is nearly flat $\Omega_{0} \sim 1$ leading to a curvature $k=0$. Combining this result with the supernova measurements one obtains that the matter content of the Universe is such that

$$
\Omega_{m} \approx 0.3, \Omega_{\Lambda} \approx 0.7
$$

It is conspicuous that this result is also corroborated by a third type of observation determining the matter density $\Omega_{m}$ alone. There are many different ways of estimating the matter density of the Universe, some of them like gravitational lensing use the property that light rays are bent by masses in general relativity. They all converge to a value of $0.1 \leq \Omega_{m} \leq 0.4$. This is enough to ensure that matter is not enough to guarantee the flatness of space-time. Another kind of matter, very different from the type of baryonic matter forming galaxies, is needed. It has been coined dark energy in contrast with cold dark matter which is responsible for the gravitational collapse leading to the formation of galaxies. Either in the form of a cosmological constant or some other type of candidates such as quintessence, dark energy represents $70 \%$ of the energy balance of the Universe.

One would like to characterise the dark energy component of the Universe. A very useful feature is the equation of state $w$ defined as the ratio between pressure and energy $p=w \rho$. The Universe comprises different types of matter of very different equations of state. Radiation has $w=\frac{1}{3}$ while Cold Dark Matter $(\mathrm{CDM})$ and ordinary dust like matter are pressure-less $w=0$. A cosmological constant is such that $w=-1$. Observation indicates that the equation of state of dark energy should be less than -0.8 , see figure 3. Exotic types of matter with a negative equation of state are favoured. In particular, as seen in figure 4, the cosmological constant is one of these possibilities. It even seems that equations of state $w<-1$ are not experimentally excluded. This would violate the energy conditions of general relativity. In particular, energy densities would not be automatically positive. This can be realized using a ghost (or phantom) scalar field with a negative kinetic energy. This type of field would be rolling towards the maxima of its potential where the equation of state would be $w=-1$. However, the excitations of the phantom field would have a negative 


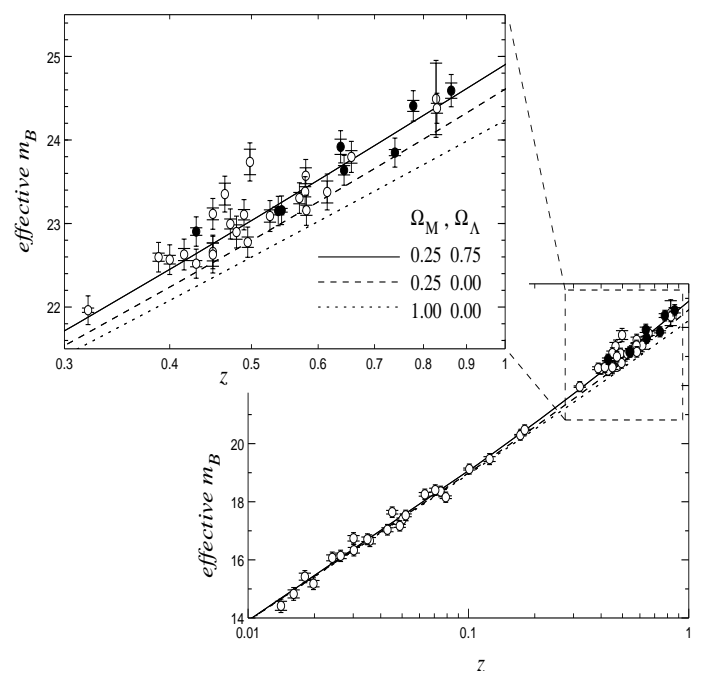

Figure 1: The Hubble diagram giving the effective magnitude of type Ia supernovae up to a red-shift of 1 . The blown up region indicates three possible scenarios for $\Omega_{m}$ and $\Omega_{\Lambda}$. The fit with the continuous line corresponds to a spatially flat Universe with $\Omega_{\wedge}=0.75$ (taken from $\lceil 61$ ).

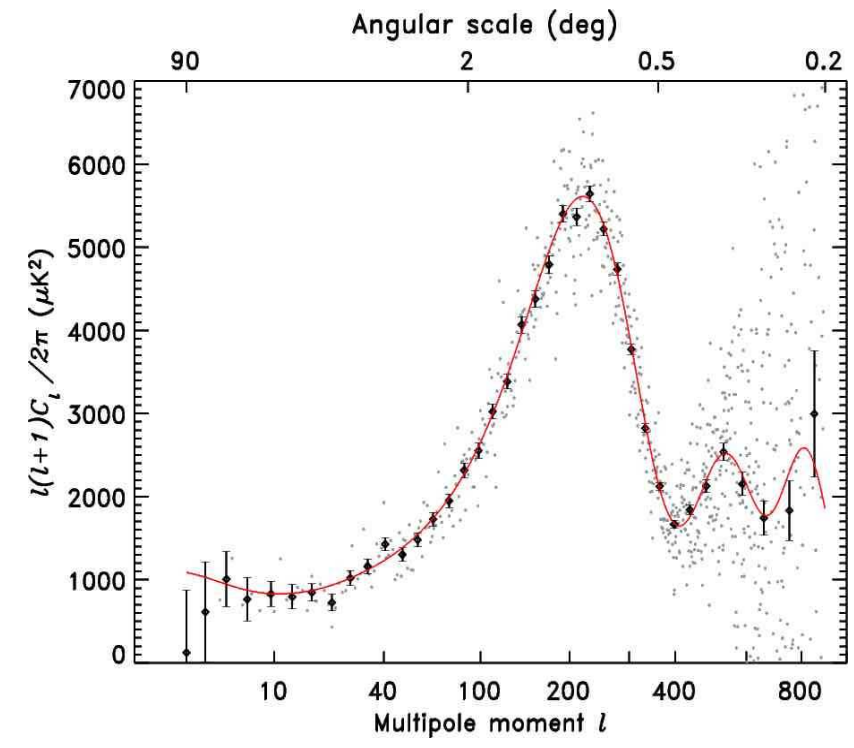

Figure 2: The WMAP result for the $C_{l}$ 's as defined in the text. Notice the location of the first peak which indicates that the Universe is spatially flat (taken from [7]). 
mass jeopardizing the stability of space-time. Empty space-time could decay into ordinary particles and phantoms. Hence such models lead to very serious problems which make them highly contrived. In the following we will examine alternatives to a pure cosmological constant with an equation state $0>w>-1$

\section{Particle Physics and the Cosmological Con- stant}

The cosmological constant problem springs from the blatant mismatch between the typical energy scales of particle physics, and therefore of all the physical phenomena taking place in the early Universe, and the energy scale $\Omega_{\Lambda} \rho_{c}$. It is $\rho_{c} \approx 10^{-48} \mathrm{GeV}^{4}$, i.e. an energy scale of order $10^{-3} \mathrm{eV}$. Recall that the mass of the electron is $m_{e} \approx 0.5 \mathrm{MeV}$ while the mass of the proton and the neutron is close to $m_{p} \approx 1 \mathrm{GeV}$. Already the critical energy density of the Universe is twelve orders of magnitude lower than the typical scale of nuclear physics $\Omega_{\Lambda} \rho_{c}<<m_{p}^{4} \approx 1 \mathrm{GeV}^{4}$. To have a better understanding of the chasm that separates the observed cosmological constant and particle physics scales, a few notions of high energy physics must be recalled.

Particle physics has fulfilled an impressive unification programme. By looking at shorter and shorter distance scales, the laws of physics simplify drastically reaching a climax with the electro-weak unification as predicted by the Weinberg-Salam model and tested to high precision at accelerators such as LEP at CERN in Geneva. Going up in energy, the electron-volt is the atomic physics scale, while the proton mass $m_{p} \approx 1 \mathrm{GeV}$ is the nuclear physics scale. Above the mass of the $W$ and $Z$ bosons of order $90 \mathrm{GeV}$, the weak force responsible for radioactivity and electromagnetism become a single electro-weak force. The scale of $100 \mathrm{GeV}$ is the current energy barrier beyond which particle physics models have not been tested experimentally. The new LHC (Large Hadron Collider) accelerator at CERN which is due to operate before the end of the decade will go beyond that threshold and probe the Higgs physics. The Higgs particle is essential to the theoretical architecture of the electro-weak theory. Its detection would be a major achievement of the unification programme. Above the TeV scale $(1000 \mathrm{GeV})$, particle physicists are prone to speculation. A host of models have been proposed ranging from supersymmetry to extra dimensions. We will come back to these models later. Eventually the final energy frontier is set by the gravitational Planck energy

$$
m_{\mathrm{Pl}}=\sqrt{\frac{\hbar c}{8 \pi G_{N}}}
$$

which is $m_{\mathrm{Pl}} \approx 2.410^{18} \mathrm{GeV}$. This is the scale above which gravity is not negligible anymore at the subatomic level. Strong gravitational effects are expected which may distort our vision of space-time. This might be the beginning of the string theory realm where particles would be best described by small open and closed strings. The cosmological constant scale $10^{-12} \mathrm{GeV}$ is thirty orders of magnitude lower that the Planck scale. 


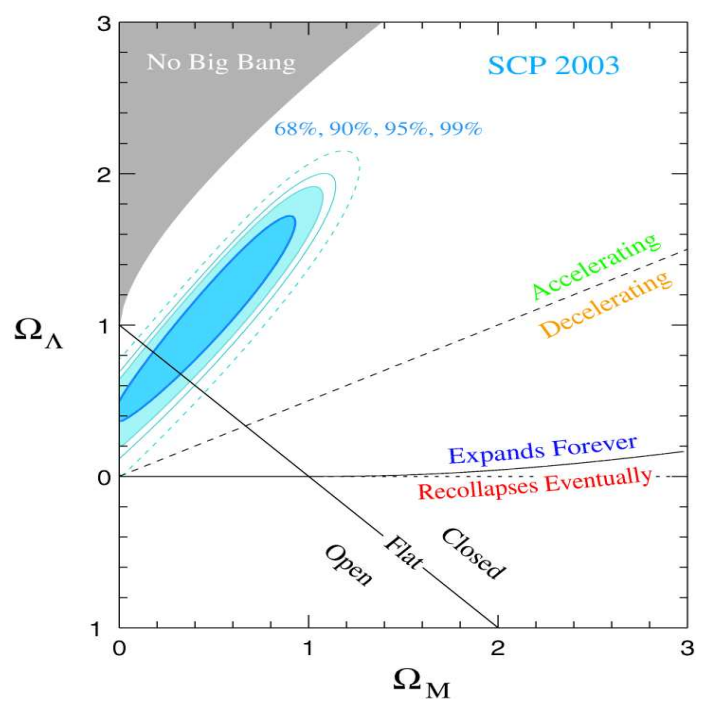

Figure 3: The contour plot for $\Omega_{m}$ and $\Omega_{\Lambda}$. The flat Universe $k=0$ constraint is indicated as a straight line and the oblong ellipses emanate from the supernovae results (taken from $[6]$ ).

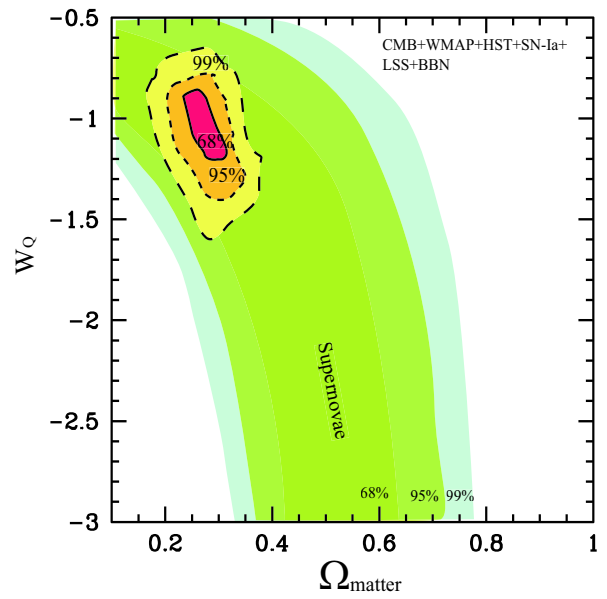

Figure 4: The combined constraints from structure formation, the cosmic microwave background and the supernovae measurements on the equation of state $w$ and $\Omega_{m}$. Notice that the contours are centered around $w=-1$, a cosmological constant. Values of $w<-1$ would violate the energy conditions and would certainly require some new physical insight (taken from [8]) 
Particle physics has a strong impact on the cosmological constant. First of all one must realize that the cosmological constant is nothing but the vacuum energy. Vacuum energy is a well-known concept in quantum mechanics. Consider the harmonic oscillator with its eigen-energies $E_{n}=\left(n+\frac{1}{2}\right) \hbar \omega$. The expectation value of the energy operator in the vacuum is $\frac{\hbar \omega}{2}$. It is not zero as one may have expected classically. In particle physics, particles are described by quantum fields corresponding to an infinite collection of harmonic oscillators. The vacuum energy associated to a particle is therefore an infinite sum $\sum_{n=0}^{n=\infty}\left(\frac{\hbar \omega}{2}\right)=\infty$. This infinite contribution is at the heart of renormalisation theory. In flat space-time where one neglects the influence of gravity, as it is the case in accelerator experiments, it makes sense to subtract an infinite contribution to the vacuum energy leaving a finite renormalised energy density. This is not physical in curved space-time where the energy density of the vacuum influences the geometry of space-time. In field theory the contribution to the vacuum energy is regularised, the infinite contribution is not physical and turns out to be $\rho_{\text {vacuum }} \propto M^{4}$ where the possible particle masses cannot exceed a certain energy scale $M$. Such an energy scale is called a cut-off. It represents the maximal energy scale for which a given particle physics model is adequate. For the electro-weak theory $M \approx 100 \mathrm{GeV}$ while models beyond the electroweak theory may be valid up to $m_{\mathrm{Pl}}$. Hence quantum fluctuations lead to a non-vanishing vacuum energy contributing to the cosmological constant.

Quantum effects up to the Planck scale give a contribution of order $m_{\mathrm{Pl}}^{4}$ to the cosmological constant. Assuming that the Universe has a bare cosmological constant $\Lambda_{0}$, the observed dark energy is then $\Lambda_{0}+m_{\mathrm{Pl}}^{4} \approx 10^{-48} \mathrm{GeV}$. This requires to adjust $\Lambda_{0}$ to 120 orders of magnitude. No physical justification to such a fine-tuning is known. Even if one could accommodate such an enormous cancellation, contributions to the vacuum energy from the electro-weak phase transition would give a contribution of order $M_{W}^{4}$ to the vacuum energy. Of course nobody knows how to cancel such disparate contributions to the dark energy. In conclusion, the vacuum energy of particle physics gives large contributions to the dark energy. All these contributions have to cancel each other somehow. In the following we will present partial attempts to tackle these issues.

\section{Supersymmetry and the Cosmological Con- stant}

One can separate the cosmological constant problem into two issues. The first one consists in understanding how the cosmological constant could be zero. The second one is concerned with generating a small vacuum energy away from the vanishing cosmological constant. In this section we will examine the possible vanishing of the cosmological constant. The following section, on quintessence, will be devoted to generating a small vacuum energy.

Exactly vanishing entities in physics are generally associated with symmetries. For instance, the gauge invariance $A_{\mu} \rightarrow A_{\mu}+\partial_{\mu} \lambda$ of QED (Quantum 
Electro-Dynamics) leads to the vanishing of the mass of the photon. This springs from the impossibility of writing a quadratic and Lorentz invariant term $A_{\mu} A^{\mu}$ in the Lagrangian of QED. Such a term would violate gauge invariance. Could it be that the cosmological constant vanishes for symmetry reason? This would be the case in a supersymmetric world.

Particles are classified according to their statistics, integer spin particles are Bosons obeying the Bose-Einstein statistics while half-integer spin particles are Fermions obeying the Fermi-Dirac statistics. Photons are Bosons while electrons are Fermions. Usual symmetries of nature such as Lorentz symmetry do not mix Bosons and Fermions, they preserve the statistics of each particle. In 1971 while trying to introduce Fermions in string theory, P. Ramond invented an unusual symmetry exchanging Bosons and Fermions: supersymmetry. Supersymmetry is a framework within which most models of particle physics can be embedded. There is for instance a supersymmetric electro-weak theory extending the electro-weak theory. How does one construct such theories? First of all supersymmetry associates to each particle another particle called a superpartner. Electrons are spin $\frac{1}{2}$ particles whose superpartners are the selectrons, spin 0 particles. Photons with spin 1 are associated to photini of spin $\frac{1}{2}$. Earlier attempts trying to identify the photino with the electron failed (they have a different charge). The supersymmetric electro-weak theory has twice as many particles as the electro-weak theory.

Another conspicuous feature of supersymmetry is its link to the Poincaré group. The Poincaré group comprises the Lorentz group, i.e. rotations and Lorentz boosts, and the translation group. When transforming a particle into its superpartner and then back into the original particle, the particle is translated in space-time. This fundamental property of supersymmetry is at the origin of its special link with the vacuum energy of the Universe. Indeed denoting the generators of supersymmetry by $Q$, they satisfy $Q \bar{Q}+\bar{Q} Q=2 E$ in the rest frame of a massive particle of energy $E$. This is the mathematical formulation of the fact that squaring a supersymmetry transformation amounts to a translation. Taking the expectation value of the left hand side in a given normalised state of the theory $\mid \phi>$ gives $|Q| \phi>\left.\right|^{2}+|\bar{Q}| \phi>\left.\right|^{2}=2 E$ from which we deduce that $E \geq 0$ the energy is always positive in a supersymmetric theory. Moreover the supersymmetric vacuum $\mid 0>$ such that $Q|0>=\bar{Q}| 0>=0$ has vanishing energy $E_{\text {vacuum }}=0$ Hence the vacuum energy of a supersymmetric theory is protected and vanishes. Such an astonishing result would provide a solution to the cosmological constant problem but for a hitch: the Universe is not supersymmetric. Indeed the superpartners have never been observed. If they exist at all, the most likely explanation for their non-observation would be a large mass splitting within supersymmetric multiplets. This requires to break supersymmetry.

The role of supersymmetry in alleviating the cosmological constant problem requires to incorporate gravity in the supersymmetry framework. This is realized within supergravity where ordinary particles are complemented with the gravitational multiplet. It comprises the graviton and its superpartner the gravitino. The graviton is the particle mediating gravity, in the same vein as 
photons mediate electromagnetism. It is a spin 2 particle, its superpartner the gravitino is a spin $\frac{3}{2}$ particle. Gravity being of infinite range, the graviton and the gravitino are massless particles. This is true as long as supersymmetry is not broken. In the most common supersymmetry breaking scheme, the socalled gravity mediated breaking, the gravitino becomes massive in manner akin to the way the $\mathrm{W}$ boson becomes massive in the electro-weak theory, it is the super-Higgs mechanism. As a result, the superpartners become heavier than the particles of the electro-weak theory. The mass splitting is of the order of the gravitino mass and is expected, if supersymmetry is ever to be detected, to be of order $100 \mathrm{GeV}$. A larger mass splitting would rule out supersymetry as a viable candidate of physics beyond the electro-weak theory.

In supergravity the vacuum energy is not guaranteed to vanish. Moreover as soon as supersymmetry is broken, the vacuum energy picks up a value of order $\rho_{\text {vacuum }}=m_{3 / 2}^{2} m_{\mathrm{Pl}}^{2} \approx 10^{40} \mathrm{GeV}^{4}$ where $m_{3 / 2}$ is the gravitino mass. Notice that this is a lot less than $m_{\mathrm{Pl}}^{4}$ but still much too high to provide a plausible explanation for the tiny value of the observed cosmological constant. So despite all its promises supersymmmetry alone is not enough to provide a mechanism explaining either the vanishing of the cosmological constant or its extremely small value.

\section{Quintessence}

Judging on the difficulty of obtaining a proper explanation for the almost zero value of the cosmological constant, one may attempt to treat the cosmological constant in a two-pronged approach. First postulate that some unknown mechanism is at play and leads to a vanishing cosmological constant. Then adjust the small amount of dark energy using four dimensional field theory. Of course this approach anticipates an eventual breakthrough which would explain the first step. The small vacuum energy can be modelled using scalar fields with simple potentials. This bears the name of quintessence.

Let us list some of the requirements that one may impose on quintessence models. First of all they must reproduce the vacuum energy now. In doing so, one would prefer not to fine-tune energy scales to values way below particle physics scales. Similarly if the energy of the scalar field were to dominate earlier, i.e. during nucleosynthesis, the model would lead to such a change in the Hubble rate that the delicate balance necessary to produce all the elements would be dramatically modified. In practice the scalar field energy cannot start dominating much before $z=1$. Such a coincidence problem, i.e. why the vacuum energy dominates nearly now and not in the past or even in the future is one of the unsolved puzzles of quintessence models. One would also like to obtain models such that the dynamics of the scalar field now does not depend too much on the initial conditions set in the early universe. Indeed having to set the values of field during the early Universe would be seen as an unexplainable finetuning. An explanation could be provided by the anthropic principle claiming that initial conditions must be set so that our Universe is the way it is in order 
to accommodate the existence of life on earth.

The dynamics of a scalar field mimics a perfect fluid with a pressure identified with the difference between the kinetic energy and the potential energy $V(\phi)$. The energy density is the sum of the kinetic energy and the potential energy. Quintessence relies on the possible existence of runaway potentials, i.e. decreasing potentials vanishing at infinity. Just like on a ski slope, the field $\phi$ rolls down the potential. For properly chosen potentials the value of the field and its kinetic energy can be arranged in such a way that the energy density is $70 \%$ of the critical energy density of the universe now. There seems to be an infinite choice of rolling potentials leading to this property. In fact, one may restrict this infinity by requiring the existence of an attractor, i.e. a long time solution of the scalar field equations of motions attracting all possible solutions. Of course the advantage of attractors is to get rid of the dependence on initial conditions. The scalar fields eventually follows the attractor, independently of the initial conditions. Attractors do exist as long as the potential decreases steeply enough. We will discuss two explicit families of potentials: exponential potentials and inverse power law potentials. Choosing the potential to be $V(\phi)=V_{0} e^{-\lambda \frac{\phi}{m_{\mathrm{P} 1}}}$, the field rolls down mimicking a fluid of constant equation of state $\omega_{\phi}=\frac{\lambda^{2}}{3}-1$. Hence it leads to an equation of state close to -1 for small $\lambda$. Moreover, in the presence of dust-like matter, one can show that the scalar field eventually dominates provided its equation of state is negative. In that case it is also a late time attractor. Hence for small $\lambda$ the exponential model provides an interesting quintessence model. Now it does not alleviate the coincidence problem. One must tune the value of the field now so that it is marginally dominant, in other words our Universe would not have reached the late time attractor and would be trawling in a transient state.

To remedy this problem one may invoke models where the attractor exists in the past. Consider the potential $V(\phi)=\frac{M^{4+\alpha}}{\phi^{\alpha}}$ where $\alpha$ is positive. As long as the scalar field is subdominant one can find an attractor where the scalar field energy density decreases slower than the matter density, implying that the scalar field density overcomes the matter density eventually. This happens as soon as $\phi$ reaches a value of order $m_{\mathrm{Pl}}$. After that the scalar field rolls slowly $\phi \approx m_{\mathrm{Pl}}$. This allows to reformulate the coincidence problem: the scalar field dominates in the recent past provided $V\left(m_{\mathrm{Pl}}\right)$ is of the order of the critical density now. This necessitates to adjust the value of $M$. For instance if $\alpha=6$, this leads to $M \sim 10^{6} \mathrm{GeV}$, a scale which is compatible with high energy physics. Of course, the value of the energy scale $M$ has to be fine-tuned. This is a pity as no clear explanation for both the value of the parameter $\alpha$ and the energy scale $M$ can be provided.

These models suffer from a serious problem, the value of the field is so large that it is difficult to justify a theory involving a single scalar field. One may invoke supergravity whose description up to the Planck scale is more justified. In that case, the supergravity equivalent of the inverse power law potential is modified $V(\phi)=\frac{M^{4+\alpha}}{\phi^{\alpha}} e^{\frac{\phi^{2}}{m_{\mathrm{Pl}}^{2}}}$. This potential leads to no modification in the 


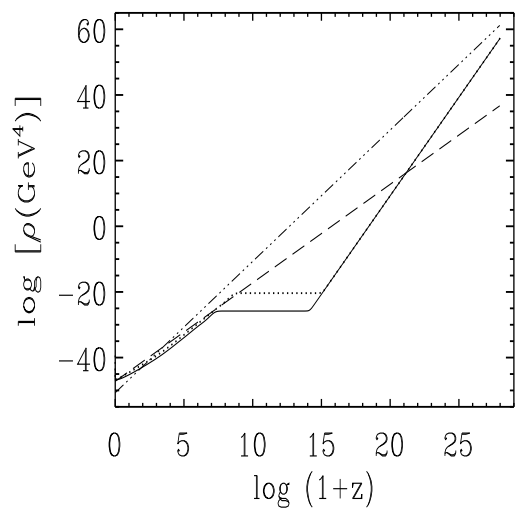

Figure 5: The energy densities of matter (middle curve), radiation (top curve) and quintessence (bottom curves for the inverse power model and its supergravity version) as a function of the red-shift. It illustrates the coincidence problem: why do the energy densities turn out to be so close now and in the recent past? (taken from [9])

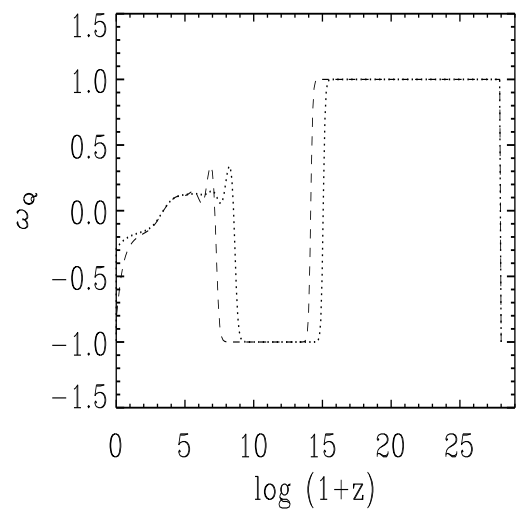

Figure 6: The evolution of the equation of state for the inverse power law potential and the supergravity corrected power law potential. The equation of state is closer to -1 now for the supergravity modified potential. Notice the frequent variations of the equation of state (taken from [9]). 
early Universe. As soon as the scalar density dominates, the potential becomes larger than the inverse power law case, hence an equation of state closer to -1 is obtained. This type of potential can be used phenomenologically as a benchmark for testing the observability of quintessence models.

Another remarkable feature of quintessence is the extreme lightness of the quintessence field with a mass of the order of the Hubble rate now $H_{0} \sim$ $10^{-43} \mathrm{GeV}$ For all practical purpose, the quintessence field can be considered to be massless leading to strong deviations from general relativity. The only possible wayout is to impose that the quintessence field is almost decoupled from known particles. This puts severe constraints and jeopadises the naturality of possible models. Similarly there is no symmetry preventing a direct coupling between the quintessence field and the kinetic terms of the photon field in QED. This leads to large variations of the fine structure constant, in contradiction with known bounds.

Other types of potentials have been presented in the last few years. As long as a comprehensive theory describing the dynamics and the origin of the quintessence scalar field is not obtained, all quintessence models only give a partial understanding of the value of the vacuum energy now.

\section{Extra-Dimensions and the Cosmological Con- stant}

So after all it seems that a satisfactory explanation for the cosmological constant problem may be out of reach. This is backed up by a no-go theorem proven by S. Weinberg and stating that the vanishing of the vacuum energy cannot be solved with field theories involving a finite number of scalar fields. Of course such theorems can always be circumvented by applying methods not covered by their premises. A possible explanation may come from the putative existence of extra-dimensions. The first model with one extra-dimension was proposed in the 1920s by Kaluza and Klein in an attempt to unify gravity and electromagnetism. Since then the number of extra-dimensions has been gradually increasing. In particular, string theory predicts that the highest number of dimensions is 11. This is obtained from the existence of an unique supergravity in 11 dimensions accommodating no particle of spin higher than 2. By compactifying one of these dimensions on a small circle, one obtains the $10 \mathrm{di}$ mensional string theories. In string theory, there exists extended objects called $p$-branes of dimension $p+1$. They represent higher dimensional object generalizing point particles $p=0$ and strings $p=1$. In particular, one popular model consists in imagining that the known particles, the strong, weak and electromagnetic forces reside on a 3 -brane. Only gravity is allowed to propagate outside the brane, in the bulk. The simplest configuration is 11 dimensional with two boundary branes, $p=9$, separated by a finite interval. One of the boundaries carries the electro-weak and strong interactions. By compactifying 6 of the 7 extra-dimensions on a manifold called a Calabi-Yau manifold, one gets a five- 
dimensional model where the extra-dimension is an interval. Compactification is a process whereby some space dimensions are curled up and of small size. To a good approximation, space-time appears to have fewer dimensions, e.g. one can go from 11 to 5 dimensions by curling up 6 dimensions. A Calabi-Yau manifold is a 6-dimensional space with vanishing Ricci tensor.

This situation has been drastically simplified by Randall and Sundrum where the bulk interval carries a negative cosmological constant and the two boundary branes have opposite tensions related to the square root of the bulk cosmological constant. The tension of a brane is similar to the tension of a soap bubble preventing its collapse under gravity.

The equations of general relativity in five dimensions have solutions where the brane configuration presents flat boundaries, i.e. the metric on the boundaries is invariant under the Poincaré group. This invariance guarantees the vanishing of cosmological constant in four dimensions as seen by an observer on the boundary branes. So this would provide an extra-dimensional explanation to the vanishing of the cosmological constant. Is it devoid of any fine-tuning? Of course the answer is no. If one modifies the delicate balance between the boundary tension and the bulk cosmological constant, one finds that an observer on the brane would identify two situations. If the brane tensions are increased, adding some energy to the system, the effective cosmological constant is positive. The acceleration of the Universe appears to be due to the increase of the energy on the branes. If one decreases the tensions, the effective cosmological constant would be negative. Of course one could fine-tune the small increase of the brane tensions. This would just be a rephrasing of the four dimensional cosmological constant problem. In the same fashion, one may incorporate more degrees of freedom in the bulk. A likely candidate is a bulk scalar field. In that case, one obtains exponential potentials of the quintessence type with a low value of the coupling constant $\lambda$. Unfortunately these models do not allay the cosmological fine-tuning problem as the value of the scalar field now has to be adjusted. Nevertheless, higher dimensional models provide an interesting framework wherein one may either reformulate or even propose new mechanisms whose four dimensional justification would have been debatable.

This is the case of self-tuning of the cosmological constant which is a very stimulating possibility. The idea can be formulated in very simple terms. The cosmological constant or the vacuum energy is a source of curvature for the four dimensional dynamics. Could it be that the same vacuum energy on our Universe seen as a 3-brane in a multi-dimensional Universe would curve the extra-dimensions while preserving the flatness of our Universe? As a result, any modification to the vacuum energy, for instance from phase transitions, would not affect the four dimensional dynamics at all. It would simply modify the geometry of unobservable dimensions.

An explicit model can be easily constructed. Consider a free scalar field living in the bulk and coupled exponentially to a four-dimensional brane. The induced geometry on the brane is flat, i.e. the Hubble rate vanishes while the bulk metric is curved with an amount depending onthe brane tension. The metric in the bulk develops a singularity at finite distance where the curvature 
of five dimensional space-time blows up. The existence of this singularity is a nuisance which may jeopardize the model altogether. In particular, shielding the singularity requires to include a brane at the singularity whose tension has to be fine-tuned to match the tension of the observable brane. The absence of fine-tuning on the observable brane has been replaced by a worse sin: one must fine-tune tensions on branes situated at different points along the fifth dimension.

After all, it may happen that gravity itself needs to be altered. One may modify gravity in five dimensions in order to smooth out the singularity. This is achieved by adding a so-called Gauss-Bonnet term in the Lagrangian of gravity. Such a correction to gravity is suggested by string theory. It arises as soon as quantum corrections to gravity are taken into account. It also turns out that Lovelock theorem guarantees that it is the only possible modification of gravity which preserves the fact that the Einstein equations are second order differential equations. Now the metric in the bulk is non-singular. The price to pay is the need to fine-tune parameters once again. Hence it seems that requiring that no singularity occurs in the bulk is connected to fine-tuning of some parameters, while the absence of fine-tuning is linked to the presence of a singularity in the bulk.

Could it be that supersymmetry in five dimensions solve the cosmological constant problem? It is indeed the case that five dimensional supergravity leads to a vanishing effective cosmological constant on the brane. The only snag is that the necessary step of supersymmetry breaking reintroduces a fine-tuning of the non-zero vacuum energy.

More generally, can one envisage to obtain de Sitter space with a small cosmological constant from the compactification of a higher dimensional supergravity theory? It turns that the Gibbons-Maldacena-Nunez theorem implies that this cannot happen if one compactifies on a static manifold with no boundary. As soon as one allows compactifications on time-dependent spaces, one may obtain de Sitter space. This is only the tip of a very active branch of high energy physics where one tries to generate de Sitter space. This is a subject still in its infancy which may provide some insight into the cosmological constant problem.

\section{Conclusion}

We have presented a few aspects of the cosmological constant puzzle. The fact that no physical mechanism providing even a qualitative understanding has been discovered, despite the efforts of numerous physicists, may call for a complete reappraisal. It could be that drastic measures such as modifications of gravity may be necessary. At present, all these models are at best contrived. A more surprising outcome, which may be hinted by observations, is that the acceleration of the Universe could be due to a cosmological constant. In this introductory review we have presented some promising mechanisms, within high energy physics, using either supersymmetry or extra-dimensions. 


\section{Further Reading:}

[1] P. Binetruy, "Cosmological Constant vs. Quintessence", lectures at Les Houches summer school "The Early Universe" and Peyresq 4, hep-ph/0005037.

[2] S. Carroll, "Why is the Universe Accelerating?", contribution to measuring and modelling the Universe, Carnegie Observatories Astrophysics Series Vol. 2, ed. W. L. Freedman, astro-ph/0310342.

[3] S. Carroll, "The Cosmological Constant", Living Rev. Rel. 4 (2001) 1, astro-ph/0004075.

[4] T. Padmanabhan, "Cosmological Constant- the Weight of the Vacuum", Phys. Rep. 380 (2003) 235, hep-th/0212290.

[5] P. J. E. Peebles and B. Ratra, "The Cosmological Constant and Dark Energy", Rev. Mod. Phys. 75 (2003) 559, astro-ph/0207347.

\section{References:}

[6] R. A. Knop et al. (the Supernova Cosmology Project), astro-ph/0309368.

[7] D. N. Spergel et al., Astro. Phys. J. Suppl. 148 (2003) 175.

[8] A. Melchiorri, L. Mersini, C. J. Odman and M. Trodden, Phys. Rev D68 (2003), 043509.

[9] P. Brax and J. Martin, Phys. Lett. B468 (1999). 40. 